\title{
La política urbana en Madrid: un relato provisional
}

\author{
Rosa DE LA FUENTE FERNÁNDEZ \\ Departamento de Ciencia Política y de la Administración III \\ Universidad Complutense de Madrid \\ rdelafuente@cps.ucm.es \\ María VELASCO GONZÁLEZ \\ Departamento de Ciencia Política y de la Administración II \\ Universidad Complutense de Madrid \\ maria.velasco@cps.ucm.es
}

Recibido: 28-05-12

Aceptado: 22-09-12

\section{RESUMEN}

El artículo reflexiona sobre las diferentes etapas por las que ha transcurrido la política urbana de la ciudad de Madrid, tratando de delimitar fases que presenten coherencia interna respecto del modelo de ciudad que se persigue y las acciones que se impulsan. La descripción de acciones se completa con una reflexión sobre las claves que permitirían entender las diferencias y similitudes en el modelo de ciudad que plantean distintos gobiernos que pertenecen al mismo partido político. Así se consideran diversos factores: si la política urbana se concentra en la lógica interna de la propia ciudad o se orienta hacia una lógica global; si se considera la ciudad como un espacio político o como un espacio de oportunidades económicas o si el protagonismo o liderazgo de las distintas fases es local o de otros niveles de gobierno.

Palabras clave: política urbana; transformaciones urbanas y política; local; global; Madrid.

\section{Urban policy in Madrid: a provisional analysis}

\begin{abstract}
Different stages of urban policies in Madris is our central reflexion of this article, trying to identify periods of internal coherence according to the city model pursuit and the actions developed. The description of actions is completed with an analysis of main key points in order to understand the difference and similitudes of the city model implemented by different governments even from the same party. Therefore, different factors are taken into account: if urban policiy is centered in the internal logic of the city, or if by contrary is oriented to a global context; if city is thought as a political space or mainly as a place for beneficts, or if the leadership of the different stages is local or is belongs to other government levels.
\end{abstract}

Key words: urban policy; urban transformations and politics; local; global; Madrid. 


\title{
A política urbana em Madri: um relato provisório
}

\begin{abstract}
RESUMO
$\mathrm{O}$ artigo reflexiona sobre as diferentes etapas pelas quais transcorreu a política urbana da cidade de Madri, buscando delimitar fases que apresentem coerência interna face ao modelo de cidade buscado e as ações impulsionadas. A descrição das ações completa-se com uma reflexão sobre as principais dimensões que permitiriam entender as diferenças e similaridades no modelo de cidade defendido por governos diferentes pertencentes ao mesmo partido político. Consideram-se, desse modo, os seguintes fatores: se a política urbana concentra-se na lógica interna da própria cidade ou se orienta à uma lógica global; se a cidade é considerada como um espaço político ou como um espaço de oportunidades econômicas; ou se o protagonismo ou liderança das diversas fases é local ou de outros níveis de governo.
\end{abstract}

Palavras chave: política urbana; transformações urbanas e política; local; global; Madri.

\section{REFERENCIA NORMALIZADA}

Fuente Fernández, Rosa de la, y Velasco González, María (2012) "La política urbana en Madrid: un relato provisional". Geopolítica(s). Revista de estudios sobre espacio y poder, vol. 3, núm. 1, 35-59.

SUMARIO: Introducción. 1. Observando las políticas urbanas. 2. Las políticas urbanas de la ciudad de Madrid. 2.1. Franquismo (1944-1979). 2.2. Primeros gobiernos democráticos (1979-1988). 2.3. Ciudad gerencial (1989-2003). 2.4. Ciudad global (2003-2011). 3. La política urbana en la ciudad de Madrid: claves para su interpretación. 3.1. Madrid en tránsito hacia la ciudad global. 3.2. El fortalecimiento de las políticas neoliberales de Madrid. 3.3. Las contradicciones de la capitalidad. 3.4. Contrapesos en los liderazgos de Madrid. Conclusiones y algunas preguntas para el futuro. Bibliografía.

\section{Introducción}

Madrid ha cambiado profundamente en los últimos cincuenta años. La ciudad de los años 1960 se ha modificado en todas sus dimensiones y, a pesar de ello, no hay muchos trabajos que reflexionen sobre la voluntad que ha determinado el camino seguido, sobre cuáles han sido las decisiones que han convertido a la ciudad en lo que hoy es, sobre la política urbana de Madrid.

Sin embargo, las investigaciones sobre la cuestión urbana y las políticas urbanas han crecido significativamente en las últimas décadas. Se han multiplicado las propuestas sobre política urbana y los estudios de caso, generando un campo rico y variado que nos permite observar la acción pública de cualquier ciudad desde posiciones diversas.

El presente artículo reflexiona sobre la política urbana de la ciudad de Madrid desde una doble premisa: la política urbana es más que la política urbanística y la política urbana es más que el conjunto de decisiones del nivel local. El objetivo del trabajo es encontrar algunas claves que permitan interpretar lo acontecido en la 
ciudad desde una perspectiva amplia, localizar las decisiones políticas de mayor impacto y concluir sobre cuál ha sido el sentido de la política para Madrid ${ }^{1}$.

\section{Observando las políticas urbanas}

Al igual que ocurre con muchos otros fenómenos sociales la política urbana se enfrenta a una dificultad inicial ligada a los problemas para su definición. En este caso la indeterminación tiene un doble origen. Por un lado, la complejidad está ligada al concepto de "cuestión urbana", expresión que hace referencia a un proceso social concreto y, al tiempo, a una categoría territorial definida (Brugué y Gomá, 1998). Por otro, también el concepto de políticas públicas contiene referencias múltiples, siendo a la vez una propuesta de acciones, un proceso político y un proceso de discusión social (Parsons, 1995).

A la condición polisémica de la expresión política urbana, se suma el hecho de ser un fenómeno de interés para disciplinas diversas. Lo que conlleva una gran variedad de enfoques, planteamientos y propuestas teóricas. Estos distintos enfoques aportan visiones complementarias que pueden tener una mayor o menor fuerza explicativa según el contexto y los casos. Pero, más allá de esta variedad de aproximaciones teóricas y análisis de casos, existe cierto acuerdo en la importancia para las ciudades en las últimas décadas de dos factores intensamente relacionados: la globalización y la extensión hegemónica de políticas y prácticas neoliberales.

Ante el fenómeno de la globalización encontramos dos posturas teóricas diferenciadas. Por un lado, hay autores que consideran que el fenómeno supone la homogenización urbana, ya que se extienden las exigencias sobre las funciones de las ciudades por todo el planeta de manera uniforme. Se trata de una revisión de los estudios clásicos sobre jerarquía y función de las ciudades, en los que ha cambiado la referencia de un sistema urbano nacional, a un sistema urbano planetario. Los estudios plantean diferentes "índices" para la propuesta de jerarquización de las ciudades en el planeta: número de sedes de las grandes compañías del plantea, fortaleza de los servicios financieros y comerciales o número de centros de innovación tecnológica, entre otros (Friedmann y Wolf, 1982; Sassen, 1991; Castells, 1989; Goodfrey y Zhou, 1999).

Otra línea de trabajo considera que existe diferencia entre la economía globalizada -y sus exigencias- y las ciudades globales. El concepto de ciudades globales está ligado a la extensión de determinados modelos y prácticas urbanas. Esta circulación a escala global de políticas urbanas exitosas despegó, según Crot (2010:

\footnotetext{
${ }^{1}$ Queremos agradecer los comentarios de los evaluadores, que han servido para mejorar el trabajo y plantearnos dudas que abren nuevos interrogantes.
} 
120), a principios de los años 1990. En un primer momento, los modelos de políticas fueron implantados desde las ciudades industriales a las ciudades de países en desarrollo, a través de las agencias multilaterales. En la actualidad podemos hablar de la existencia de un mercado de intercambio multilateral, donde experiencias latinoamericanas y asiáticas son proclamadas como soluciones innovadoras y aplicadas en contextos europeos, fundamentalmente. Así, la política urbana habría adquirido una relevancia cada vez mayor, en todas las escalas de gobierno, de manera que la dimensión urbana se ha ido convirtiendo en un eje central de la vida política y de las políticas pero, al mismo tiempo, las propuestas de acción pública serían cada vez más homogéneas.

Teóricamente la política urbana, según propuestas de organismos internacionales y gobiernos locales, tendría dos objetivos:

-Un objetivo exógeno: convertirse en lugares "atractivos" para poder ascender en la escala de ciudades globales (a través de grandes reformas morfológicas para sustituir los espacios producidos durante la fase de producción fordista, planes estratégicos de internacionalización de las ciudades, arquitectura icónica, cambios en el planeamiento urbano, etc.).

- Un objetivo endógeno: convertirse en ciudades que destaquen por ser ciudades con calidad de vida ambiental y cultural, a partir del fomento de la participación ciudadana y el sentimiento de comunidad (por medio de planes participativos de gestión del espacio público, Agendas 21, etc.).

Esta difusión de prácticas urbanas exitosas ha supuesto la incorporación sistemática de los siguientes temas en la mayor parte de las agendas de gobierno urbano: desarrollo sostenible; modernización, tecnología y productividad; equidad y eficiencia de la planificación; operaciones público-privadas; multiculturalismo; memoria urbana; renovación de áreas, y gobernanza y participación (Vainer, 2000).

Tanto si es consecuencia de la globalización y extensión de exigencias comunes, o a la difusión de agendas, esta creciente homogeneización planteó la cuestión de la pérdida de importancia de la ciudad en el siglo XXI. En el debate actual las posturas más aceptadas señalan que la supuesta desterritorialización no ha ocurrido, más bien ha sucedido lo contrario, aumentando la importancia del lugar y de las localidades (Castells, 2002; Swyngedouw, 2004; Brenner, 2003). Así lo explica Saskia Sassen (1998: 3):

Más que volverse obsoletas debido a la dispersión detonada por las tecnologías de información, las ciudades: a) concentran funciones de comando; b) son sitios de producción postindustrial para las industrias líderes de este período, financieras y de servicios especializados; y c) son mercados transnacionales donde las empresas y los gobiernos pueden comprar instrumentos financieros y servicios especializados. 
En relación con el segundo de los factores, toda la literatura señala el giro neoliberal de las políticas económicas y de la cultura política en general que se produce a partir de la década de los ochenta del siglo XX como una de las claves básicas para interpretar la política urbana a partir de entonces (Harvey, 2005; Cochrane, 2007). Desde ese momento el paradigma socio-económico keynesiano y el proyecto político afín que tenía como horizonte el desarrollo y el bienestar social se rompe y se extiende la "ciudad única" con dinámicas comunes: se priman las estrategias de eficiencia y competitividad; se impone el objetivo de convertirse en nodo de acumulación (especialmente inmobiliaria); se afinan la exclusión y clasificación espacial, y se van sustituyendo los planes, como instrumentos de intervención, por estrategias sectoriales y proyectos fragmentarios (De Santiago, 2007).

Cuadro 1. Estructura tipo de la oferta de políticas locales

\begin{tabular}{|c|c|}
\hline \multicolumn{2}{|c|}{$\begin{array}{l}\text { Políticas de promoción económico local } \\
\text { Iniciativas de desarrollo económico }\end{array}$} \\
\hline Políticas de promoción del tejido empresarial & Políticas de desarrollo territorial \\
\hline $\begin{array}{l}\text { - Innovación } \\
\text { - Atracción }\end{array}$ & $\begin{array}{ll}\text { - } & \text { Centralidades } \\
\text { - } & \text { Infraestructuras }\end{array}$ \\
\hline Políticas de empleo y recursos humanos & Políticas de desarrollo comunitario \\
\hline $\begin{array}{l}\text { - Formación } \\
\text { - }\end{array}$ & $\begin{array}{ll}\text { - } & \text { Cooperación } \\
\text { - } & \text { Servicios }\end{array}$ \\
\hline \multicolumn{2}{|c|}{$\begin{array}{l}\text { Políticas locales de bienestar social } \\
\text { Programa de servicios personales }\end{array}$} \\
\hline $\begin{array}{l}\text { Políticas sociosanitarias } \\
\text { Acción contra la exclusión }\end{array}$ & $\begin{array}{c}\text { Políticas socioculturales } \\
\text { Dinamización comunitaria }\end{array}$ \\
\hline $\begin{array}{ll}\text { - } & \text { Servicios sociales } \\
\text { - } & \text { Salud } \\
\text { - } & \text { Consumo }\end{array}$ & $\begin{array}{ll}\text { - } & \text { Cultura } \\
\text { - } & \text { Educación } \\
\text { - } & \text { Juventud }\end{array}$ \\
\hline \multicolumn{2}{|c|}{ Políticas urbanas y de territorio } \\
\hline Politicas urbanas de la eficiencia & Políticas urbanas de la equidad \\
\hline $\begin{array}{l}\text { - } \quad \text { Centralidades } \\
\text { - Infraestructuras }\end{array}$ & $\begin{array}{ll}\text { - } & \text { Vivienda } \\
\text { - } & \text { Equipamiento } \\
\text { - } & \text { Transporte }\end{array}$ \\
\hline
\end{tabular}

Fuente: Brugué y Gomá (1998: 33).

La ciudad se considera desde la lógica de la competitividad y el mercado y los actores públicos lideran una profunda acción desde el discurso de la retirada del ámbito público con características singulares (Larner, 2000; Peck y Tickell, 2002). A estos dos factores podemos sumar un tercer elemento: el incremento generalizado de la acción pública a nivel local y su progresiva complejidad. Según Saunders (1986), en la construcción de los Estados de bienestar europeos, tras la Segunda 
Guerra Mundial y durante casi tres décadas, los Estados centrales se centraron en la esfera de la producción, mientras que los actores locales se concentraron en políticas sociales, incorporando posiciones pluralistas de actores e intereses. Brugué y Gomá (1998) analizan cómo estas agendas locales, relativamente simples, se han ido densificando, incorporando acciones relacionadas con la dimensión económicolaboral de sus municipios, con la dimensión sociocultural y con nuevos modelos urbanos-territoriales (Cuadro 1). Desde los años 1990, ante la nueva complejidad de los espacios urbanos, los gobiernos locales han de responder a muchas más demandas diseñando e implantando propuestas múltiples y se ven obligados a asumir roles más estratégicos y cualitativos, convirtiendo al territorio en el eje de su proyecto económico. En este contexto globalizado, dominado por una agenda hegemónica y con un grado alto de complejidad, se desenvuelve la política urbana y, por tanto, la política de la ciudad de Madrid.

Para su análisis consideramos que una política pública es el cauce de acción impulsado por un gobierno legítimamente elegido -en ocasiones junto con otros actores no gubernamentales, especialmente movimientos vecinales y sociales- con el fin de alcanzar diferentes objetivos relacionados con un sector o un espacio territorial concreto ${ }^{2}$. La naturaleza del fenómeno sobre el que se pretende intervenir, en este caso la ciudad, es el punto de partida para determinar qué dimensiones lo conforman y qué ámbitos de acción concreta son parte de la política analizada.

En relación con lo anterior, consideramos la política urbana de Madrid como el conjunto de actuaciones que han sido impulsadas por el gobierno de la ciudad o, en su caso, por niveles de gobierno superiores en el ámbito de su competencia -en ocasiones en colaboración con otros actores no gubernamentales- con el fin de alcanzar diferentes objetivos urbanísticos, económicos, sociales y culturales para la ciudad. A ello dedicaremos el siguiente epígrafe.

\section{Las políticas urbanas de la ciudad de Madrid}

Madrid ha cambiado profundamente en los últimos sesenta años. La ciudad ha crecido a un ritmo sostenido; se han multiplicado sus infraestructuras de comunicación, zonas verdes, equipamientos educativos, sanitarios y culturales. El transporte urbano se ha transformado sustancialmente y su estructura productiva se ha terciarizado. Algunas zonas han mejorado su posición y otras aún sufren graves problemas.

\footnotetext{
${ }^{2}$ No existe una definición consensuada de política pública, al contrario, existen muchas que ponen el acento en diferentes aspectos de este espacio de la realidad política. Nuestra propuesta se construye con las definiciones de Jenkins (1978); Mény y Thoenig (1992) y Subirats et al. (2008).
} 
Su población ha variado radicalmente en cuanto a diversidad cultural, enriqueciendo su carácter y planteando retos completamente desconocidos.

Parece razonable considerar que este cambio también ha supuesto una evolución igualmente profunda en el gobierno de la ciudad, pero hay poca investigación sobre la política urbana de la ciudad ${ }^{3}$, en especial, sobre aspectos que consideramos claves para la reflexión sobre ésta.

Nuestro objetivo, en un estudio de carácter exploratorio, es construir un relato descriptivo de la acción pública que abarque un periodo de tiempo amplio, detecte aquellas acciones que han resultado las más significativas para los ciudadanos o para los propios actores políticos y permita plantearse cuestiones más reveladoras respecto de la política urbana de la ciudad de Madrid.

Un trabajo anterior nos permite afirmar que es posible detectar cuatro etapas diferenciadas en la política urbana madrileña, fases que describimos brevemente ${ }^{4}$.

\subsection{Franquismo (1944-1979)}

Desde los años 1940 Madrid se convirtió en el lugar de referencia del régimen político franquista. Su diseño, contenido en el Plan Bidagor de 1944, concebía un espacio segregado entre el centro, la llamada almendra central, y la periferia. La ciudad se organizó en función de los usos del suelo y de las necesidades de reconstrucción del Gran Madrid. Siguiendo este modelo, la industria se ubicó en la periferia sur de la ciudad, mientras que el centro de la ciudad se profundizaría progresivamente la terciarización de su actividad económica.

Si nos centramos en observar el papel del gobierno local, en términos de Brenner (2003), la ciudad era una simple correa de transmisión de las necesidades de desarrollo del Estado, sin que el gobierno municipal o provincial, tuvieran ninguna relevancia como actor político. Esta primera fase puede caracterizarse como una etapa de expansión territorial y desarrollismo en un entorno tecnocrático que no está interesado sino en construir una capital útil para el régimen político del país.

\footnotetext{
${ }^{3}$ En torno a un proyecto de investigación dirigido por el profesor Subirats, un grupo de investigadores de distintas ciudades españolas comenzamos a trabajar sobre las políticas urbanas en España, buscando similitudes y diferencias y reflexionando sobre los puntos de inflexión en la tipología de políticas urbanas. Se recopiló documentación desde la transición española hasta el año 2009 con la intención de observar qué había sucedido en las distintas ciudades en diferentes dimensiones: la dimensión territorial, de gobierno, económica, en la estructura social y la sociedad civil. El resultado de dicho trabajo es la base del presente artículo y está publicado en un libro conjunto (ver Iglesias et al., 2012).

${ }^{4}$ En el presente artículo resumiremos los datos que nos permitan avanzar en el razonamiento. El lector interesado en conocer la descripción completa puede consultar la obra anteriormente señalada (Iglesias et al., 2012).
} 


\subsection{Primeros gobiernos democráticos (1979-1988)}

La segunda fase comienza con los primeros gobiernos municipales democráticos. La relocalización de la política en el ámbito local facilita el impulso de los procesos de descentralización y fortalecimiento del municipalismo, por un lado, e impulsa la movilización ciudadana, por otro. Así, al igual que ocurre en otras ciudades, el primer alcalde democrático, Tierno Galván, ejercerá un liderazgo político desde la izquierda basado en la idea de que las políticas locales permiten una mejor transmisión de las necesidades ciudadanas a las instituciones democráticas, por su cercanía. En este contexto se producen coaliciones entre el gobierno municipal y el tejido social que había generado la movilización vecinal desde los años 1960.

En esta fase domina un referente urbanístico de gran personalidad. Se persigue un modelo de ciudad compacta, que restrinja el suelo disponible a cambio de potenciar al máximo el objetivo del requilibrio interno de la ciudad. En este sentido se aprueban el Plan Especial de Protección y Conservación de Edificios HistóricoArtísticos de la villa de Madrid (1980) y el Plan General de Ordenación Urbana de Madrid (1985). Este último supuso el impulso de un nuevo modelo de ciudad, incorporando nuevos valores como la protección del medio ambiente - duplicando las zonas verdes de la ciudad-y del patrimonio edificado, así como la mejora de equipamientos sociales y culturales ${ }^{5}$, aunque fue criticado por ser extraordinariamente restrictivo en la clasificación del suelo urbanizable.

También en el plano del urbanismo se potenciaron procesos de participación vecinal en el diseño e implantación de acciones públicas, resultando algunas de gran interés, como el Programa de Barrios en Remodelación (1979-1989), que supuso la construcción de 38.000 viviendas en 29 actuaciones, una por Distrito (Planes de Actuación Inmediata).

El contexto predominante en este período será la crisis económica y el desempleo - principalmente juvenil-, en especial en las áreas periféricas de la ciudad, producto de las primeras políticas de reconversión industrial. La concentración de la vulnerabilidad social en las áreas periféricas de la ciudad, marcó la marginalidad de ciertas áreas urbanas del sur de la ciudad, estigma que aún hoy perdura. Pero este contexto no afecta por igual a todos.

\footnotetext{
${ }^{5}$ El principio básico del PGOU de 1985 fue la intervención difusa, con vocación de modificar las condiciones de toda la ciudad, mejorando la calidad en todos los distritos, en especial los que tenía mayores problemas. Esa visión inicial ha dejado un impacto profundo: "Lo específico del cambio en Madrid es que ha afectado menos al centro que a la periferia y que no se ha individualizado en unas pocas operaciones de prestigio. Ha sido una transformación generalizada, diseminada, compuesta por centenares de actuaciones de tamaño pequeño o mediano, muy repartidas por toda la geografía municipal, más atentas a la resolución de problemas concretos (de vialidad, de vivienda, de equipamiento, etc.) que a la configuración de gestos espectaculares” (López de Lucio, 2000: 110).
} 
Por un lado, entre 1975 y 1984 se destruyeron 200.000 empleos y el tejido industrial madrileño fue seriamente castigado, con cierre de empresas, reducción de plantillas y degradación del entorno industrial ante la falta de políticas efectivas de formación y recualificación de la mano de obra. Por otro lado, los nuevos servicios a las empresas (como las agencias de publicidad, las empresas de estudios de mercado, las empresas de servicios informáticos, consultorías, estudios de ingeniería, mensajerías...) crecieron en número y dimensiones a pesar de la crisis (Valenzuela, 1990). En 1985 apareció el primer polígono industrial de tecnologías avanzadas, en Tres Cantos, y se comenzaron a diseñar otros parques tecnológicos y empresariales como la Ciudad de la Imagen. El otro gran sector que experimentó un importante crecimiento durante la crisis fue el sector público, con incrementos del empleo en los tres niveles de la Administración Pública (estatal, autonómico y local).

Además, el desarrollo de la sociedad de consumo de masas introdujo nuevas demandas de productos y servicios, lo que encontró respuesta sobre todo en el ámbito de sector comercial, con la expansión de los grandes almacenes, la irrupción de las grandes superficies comerciales y la entrada de las compañías transnacionales del sector de la distribución comercial. En 1983 se inauguraba en Madrid el primer Centro Comercial Madrid-2 en La Vaguada.

En relación con las infraestructuras de transportes, el Metro deja de ser empresa privada, se acomete una primera remodelación del aeropuerto y se comienza la M-30.

Estos factores, sumados al efecto de la apertura y modernidad de la política cultural municipal, permiten la aparición de movimientos artísticos alternativos y contraculturales que convertirán a la ciudad en la sede de la llamada "movida madrileña".

El gobierno de la ciudad muestra un interés real por incorporar actores y ejercer una labor de coordinación. La ciudad se descentraliza en 18 distritos. Se aprueban las primeras Normas de Participación Ciudadana (1979) y, más adelante, los Consejos Sectoriales de participación (1988), desarrollando la estructura prevista en la LRBRL de 1985. Aunque también se reflejan las respuestas a la crisis del modelo racional burocrático y la búsqueda de una mayor eficacia a través de la creación de organismos especializados. Así se crean la Empresa Mixta de Tráfico (1982), la Empresa Municipal de Vivienda y Suelo (1987) y la Empresa Madrid Espacios y Congresos (1987).

En 1983 se crea la Comunidad Autónoma de Madrid, una nueva escala de gobierno que supone la irrupción de un nuevo actor político en el escenario de la política urbana. En el caso de Madrid, la identificación entre ambos niveles es tan intensa que en ocasiones se habla de la Comunidad Autónoma como una figura más similar a un ente metropolitano que a una unidad territorial de gobierno. De hecho, la creación de la CAM ha impedido sistemáticamente la posibilidad de desarrollar el planeamiento supramunicipal.

Desde la muerte de Tierno Galván en 1986, el gobierno de Barranco Gallardo fue incapaz de mantener las coaliciones de izquierdas, se rompieron las alianzas 
sociales, todo ello en un contexto político adverso al coincidir con el período de protesta social que llevaría a la primera huelga general al gobierno del Estado del socialista Felipe González (1988). En Madrid, el alcalde sufrió una moción de censura en 1989 que permitió que la alcaldía pasara a manos del CDS (siendo nombrado alcalde Agustín Rodríguez Sahagún) con el apoyo de los concejales de Alianza Popular. En 1991, ya bajo el nombre de Partido Popular, el partido conservador alcanza la mayoría absoluta.

En los últimos años de la etapa, bajo el mandato del Barranco Gallardo, en 1988 se crea PROMADRID. Una empresa pública del Ayuntamiento y de la Comunidad de Madrid, en la que también participan la Cámara Oficial de Comercio e Industria de Madrid, la Confederación Empresarial Independiente de Madrid y la Fundación "Amigos de Madrid", y cuyo "objetivo genérico es la promoción y desarrollo de la capital". Con este objetivo, este organismo realiza un diagnóstico que pretendía ser la base del Plan Estratégico de la Ciudad, y se publica con el título Un futuro para Madrid, una ciudad para todos. Madrid a las puertas del futuro.

\subsection{Ciudad gerencial (1989-2003)}

En 1989 un acuerdo entre el PP y el CDS, lleva a la alcaldía a Agustín Rodríguez Sahagún. En 1991 José María Álvarez del Manzano obtiene la mayoría absoluta para el PP, mayoría que revalida en 1995 y 1999, siendo el regidor que más elecciones ha ganado de forma consecutiva en la historia de Madrid.

El proceso de planificación estratégica se retoma y finalmente en 1993 se publica la propuesta con un nuevo modelo de ciudad (Cuadro 2), que ya dibuja más claramente un rol de ciudad vinculado a un objetivo de proyección externa, y en cualquier caso, siempre a través de una narrativa que busca la excelencia y la competición con otras ciudades y lugares estratégicos.

También, en esta fase se revisó el Plan General de Ordenación Urbana, incorporando cambios que permitieron un rápido crecimiento del suelo urbanizable. Se aprueban los PAUs de Montecarmelo, Las Tablas, Sanchinarro, Ensanche de Vallecas y Carabanchel, modificando la lógica de la contención del suelo del plan anterior. Mientras que a principios de los años 1990 se inicia la operación urbanística denominada Pasillo Verde Ferroviario, a partir de un Consorcio formado por el Ayuntamiento y Renfe que posibilita la ordenación y urbanización de los terrenos ocupados por las vías de ferrocarril que enlazaban antiguas estaciones de Peñuelas, Imperial, Delicias y Atocha, y la construcción de más de 1.200 viviendas. Transformación que ya se había diseñado en el plan anterior de 1985. 


\section{Cuadro 2. Visión Estratégica de Madrid en 1993}

Madrid debe aspirar a un modelo de ciudad que responda a los objetivos siguientes:

- Consolidarse como capital regional europea.

- Mantenerse como centro de toma de decisiones y centro financiero del país.

- Conducir la progresiva integración del espacio económico y social de la Península Ibérica en el sistema urbano europeo.

- Servir como proyección internacional del país.

- Convertirse en la entrada aeroportuaria en Europa para los vuelos de Iberoamérica y África.

- Emerger como el gran proveedor de servicios avanzados del Arco Mediterráneo.

- Llegar a ser un centro puntero en actividades industriales de alto valor añadido.

- Reforzarse como centro logístico de primer rango en la Europa meridional.

- Consolidarse como el corazón científico del país.

- Transformarse en un centro cultural y turístico de primer orden.

- Alcanzar una calidad de vida para sus habitantes equiparable a la de las ciudades más avanzadas del continente.

Fuente: PROMADRID (1993).

Una de las principales transformaciones será la modernización del patrimonio cultural de la almendra central que se impulsa por los tres niveles de gobierno. Serán las acciones del gobierno nacional las que posibiliten la inauguración del Museo Nacional Centro de Arte Reina Sofía; el Museo Thyssen Bornemisza; el Auditorio Nacional de Música y la Casa de América (antiguo Palacio Linares). La Comunidad Autónoma pone el marcha el Consorcio del Plan de Rehabilitación y Equipamiento de Teatros. Otras actuaciones como el Museo de la Ciudad; el Invernadero de Arganzuela, el Parque Rodríguez Sahagún; el Parque Juan Carlos I o el Faro de Iluminación y Comunicaciones de La Moncloa serán responsabilidad del Ayuntamiento. Junto a estas acciones puntuales, se aprueba un Plan de recuperación del casco antiguo, que conlleva la reforma de la Plaza de Oriente o la rehabilitación de la Gran Vía. Todo esto en el contexto de Madrid Capital Europea de la Cultura 1992.

Las demandas ciudadanas fueron parcialmente neutralizadas por la intervención activa del gobierno de la Comunidad Autónoma. Fue significativo como la inseguridad, la marginalidad, el desempleo y la vulnerabilidad de infraestructuras de comunicación y transporte de los distritos del sur de Madrid continuaron siendo los problemas que planteaba el Movimiento por la dignidad del Sur, desde Villaverde y Orcasitas. Sin embargo, el Plan de Inversiones y Actuaciones en los distritos de Usera y Villaverde se financió como una inversión extraordinaria de la Comunidad de Madrid durante seis años, entre 1998 y 2003. La participación del Ayuntamiento fue indirecta. 
En el mismo sentido, también las demandas del Movimiento por la Calidad de la Educación sur y este de Madrid que surge en 1994 por la situación de marginalidad en estos distritos, logrará pactos por la calidad de la educación con la CAM en 2001 y 2005, sin participación del Ayuntamiento.

La apuesta más significativa de la etapa es la inversión que se hace en la ciudad para convertirla en un espacio competitivo a nivel global, para lo que se intensifican los esfuerzos en infraestructura de transportes y conectividad. Y también en este caso, participan los tres niveles de gobierno.

El Plan para el Transporte en las Grandes Ciudades 1990-1993 (con fondos estructurales europeos) es el comienzo del cambio. El sistema viario arterial y radioconcéntrico comienza a dibujarse y explica la notable descentralización poblacional y de actividades. Es, así mismo, un factor básico para comprender la estructura territorial en la década de los noventa. Entre 1996 y 2003 se ejecutan diversas actuaciones. Se ejecuta el semianillo de circunvalación M-45 entre la M-40 y la M50 , que servirá de eje de distribución longitudinal este-oeste para el PAU de Vallecas y los nuevos barrios del sureste propuestos por el Plan General de 1997 (Cañaveral, Los Cerros, Los Ahijones, Valdecarros y Los Berrocales).

Esto permite que el corazón empresarial y financiero se distribuya, buscando enclaves en la fachada de las grandes autopistas (eje N-II, N-VI, M-30, etc.), o en nuevas localizaciones con mayor prestigio social (campus BSCH, etc.) y una buena accesibilidad al aeropuerto.

También el Gobierno Central aprueba el Plan Barajas que pretendía una gran renovación del aeropuerto (a partir del año 2000).

En 1987 se crea el Consorcio Regional de Transporte, como un organismo autónomo de la administración regional, con un consejo de administración en el que estarán representados, los ayuntamientos con especial mención al de Madrid, las empresas consorciadas, asociaciones patronales y sindicatos, y un Director Gerente.

También en el ámbito de la CAM se desarrolla la estrategia de Transportes regional y urbano que planea completar la línea circular del Metro (1996) y enlazar las Estaciones de Príncipe Pio y Atocha a través del Pasillo Verde Ferroviario, completándose así la denominada M-30 ferroviaria. Desde 1995-1999, la CAM hace $56 \mathrm{~km}$ nuevos de metro y 35 estaciones nuevas, puestas en servicio hasta 1998. En 1998 se amplía hacia el Aeropuerto y Recintos Feriales. Posteriormente su Plan de Ampliación planeará duplicar la Red de metro con una clara vocación de articulación metropolitana (1999-2003), en torno a Madrid, a través de la Línea 10 que llegará hasta la primera corona (Alcorcón) para conectar con METROSUR (línea circular que conecta con municipios del Sur Metropolitano). Así, la ciudad de Madrid se ve beneficiada como nodo de comunicaciones a partir de estrategias de la escala regional.

Otras actuaciones municipales con repercusión en el ámbito de la mejora de las comunicaciones turísticas, son la la inauguración de la Estación Puerta de Atocha y el primer AVE Madrid-Sevilla (1992), la Inauguración de la Estación Sur de Auto- 
buses (1997) el recinto ferial IFEMA en el Campo de las Naciones (1991) y el nuevo Palacio de Congresos (1993).

\subsection{Ciudad global (2003-2011)}

En el año 2003 Alberto Ruiz Gallardón revalida la victoria del Partido Popular, siendo reelegido en 2007 en una segunda convocatoria electoral. A pesar de pertenecer al mismo partido, el nuevo alcalde asume el gobierno de la ciudad con un liderazgo totalmente diferente. Todas las acciones emprendidas buscan dos objetivos claros: aumentar la importancia política de la ciudad, mejorando para ello sus estructuras de decisión, y profundizar en el modelo de ciudad competitiva global, interviniendo de manera clara en ámbitos claves que permitan el desarrollo de determinadas actividades económicas.

En relación con el primero de los objetivos, el Alcalde consigue que en 2006 el gobierno central apruebe la Ley de capitalidad y de régimen especial de Madrid, que busca mejorar la eficacia en la gestión administrativa y aumenta algunas de las competencias municipales en materia de movilidad, seguridad ciudadana e infraestructuras. En un plazo inferior a cuatro años revisa y somete a aprobación los estatutos de todas las entidades locales que habían sido creadas como instrumentos organizativos del ayuntamiento ${ }^{6}$. Además de impulsar la Agenda 21 Local (municipal y por distritos) desde 2005, acomete varias tareas:

La modernización tecnológica de numerosos procedimientos administrativos, su transparencia a través de Internet (incluso, con traducción al inglés de muchos documentos oficiales) y la implementación de sistemas de gestión de la calidad y de evaluación de los servicios administrativos (mediante un llamado "Sistema de Cartas de Servicios" materializado en 25 documentos hasta octubre de 2009) (Alguacil et al., 2012: 128).

\footnotetext{
${ }^{6}$ En 4 años se modifican los estatutos de las siguientes entidades: Empresa Municipal de la Vivienda y Suelo de Madrid, S.A.; O.A. Agencia de Desarrollo Económico Madrid Emprende; O.A. Agencia Tributaria de Madrid; O.A. Local Agencia para el Empleo de Madrid; O.A. Madrid Salud; O.A. Patronato de Turismo de Madrid; Organismo Autónomo Agencia Tributaria de Madrid; Sociedad Mercantil Madrid Arte y Cultura, S.A.; Sociedad Mercantil Madrid Infraestructuras Deportivas 2012; Sociedad Mercantil Madrid Movilidad, S.A.; Centro Integral de Formación de Seguridad y Emergencias; Comisión de Vigilancia y Seguimiento de la Gestión Integral de la M-30; Comisión Local de Alerta Atmosférica; Consejo de Cooperación al Desarrollo del Municipio de Madrid; Consejo Local para el Desarrollo y el Empleo de Madrid; Consejo Municipal de Consumo; Consorcio Centro de Formación de Hostelería y Restauración (San Blas); Foro de Sostenibilidad de la Ciudad de Madrid; Foro Madrid de Diálogo y Convivencia; Foro Técnico Local sobre las Personas sin Hogar en Madrid; Fundación Madrid 2012; Fundación Madrid Ciudad Global 2010; Fundación para la Movilidad y Seguridad Vial; Fundación Voluntarios por Madrid.
} 
En relación con el objetivo de crear una ciudad global, crea la Empresa Municipal Promoción Madrid (esMadrid.com), en 2002. Esta sociedad 100\% participada por el Ayuntamiento de Madrid, se dedica a la prestación de todo tipo de servicios relacionados con la promoción y difusión cultural, turística, económica y empresarial de la ciudad de Madrid a través de las nuevas tecnologías. Sin duda alguna, el énfasis en la promoción urbana estaba relacionado con la candidatura madrileña a la sede de los Juegos Olímpicos de 2012. La sociedad será la encargada también de las candidaturas para 2016 y 2020.

Unos años después, en 2007, se crea la Oficina Madrid Global, cuyo fin es "proyectar y comunicar la posición real de Madrid, mediante la realización de una serie de proyectos estratégicos de la mano de agentes clave que persiguen similares objetivos a los de Madrid Global" (Oficina de Estrategia y Acción Internacional, 2008). A diferencia de las propuestas de la fase anterior, en esta fase el marketing urbano se utiliza como herramienta para promocionar la ciudad, y por tanto en los discursos contenidos en los documentos de planificación ya no se parte de la identificación de los problemas, sino de las fortalezas que permitirán proyectar internacionalmente el "éxito" de la ciudad. Así se declara:

Madrid es la tercera metrópoli de la Unión Europea y su realidad urbana refleja los beneficios de una economía dinámica, con unas tasas de crecimiento superiores, continuadamente, a la media europea. Madrid es la puerta de entrada al mercado latinoamericanos, norteafricano y a la Unión Europea, y posee una posición cultural y geoestratégica clave como nexo natural entre esos mercados (Oficina de Estrategia y Acción Internacional, 2008: 1)

Las funciones de esta oficina estarán claramente vinculadas al reposicionamiento de la imagen de Madrid: a) análisis del Posicionamiento Internacional de Madrid: seguimiento continuado de los numerosos ranking y clasificaciones de ciudades existentes; b) participación en eventos globales, candidatura olímpica 2016, exposición universal de Shangai 2010; c) Cátedra Madrid Global de Estrategia Urbana internacional con el IE Business School; d) Colaboración con organismos internacional; e) participación en redes de ciudades, y f) diplomacia pública urbana (Oficina de Estrategia y Acción Internacional: 2-3).

En Madrid, a diferencia de otras ciudades, se ha utilizado poco la planificación estratégica desde 1993. Se aprobó un plan estratégico del Centro en 2004, aún sin desarrollar. El Plan Madrid Centro está más vinculado a la producción de la ciudad inmaterial, porque la herramienta supera el ámbito urbanístico e incorpora propuestas vinculadas a la realidad urbana, como el medio ambiente, la integración social, la movilidad, el medio ambiente, la cultura, la economía y la seguridad.

En este período, en cambio, se aprueban obras de acupuntura urbanística, sin reformar el Plan General de Ordenación Urbana, utilizando los proyectos como figuras menores de planeación urbanística. Es el caso del Proyecto de remodelación 
de la calle Serrano (2005), el Plan Remodelación Eje Recoletos-Prado o más recientemente el Plan de Renovación Urbana del entorno del Río Manzanares, también llamada "operación Río". Se impulsa la operación Chamartín (2009) y la construcción de las cuatro torres más altas de la ciudad (2004-2008) que se han convertido en un icono de la nueva forma de entender el modelo urbano. Desde 2007 en la agenda del gobierno municipal está la Cañada Real Galiana "aplicando un modelo social, para lo que los distintos ayuntamientos deberán elaborar un censo de los habitantes de la zona, que ya ha sido realizado por el Ayuntamiento de Madrid dentro de su término municipal" (Ayuntamiento de Madrid, 2012). También relacionado con la apuesta por la internacionalización, se modifica la normativa urbanística para permitir instalar hoteles en edificios de la ciudad, sin tener que tramitar para ello Planes Especiales, bastando una licencia municipal, lo que permite el cambio de uso de algunas de las principales vías de la ciudad.

Sin duda alguna, la gran obra del periodo es el soterramiento de la M-30. Para ello, se firma un Convenio entre el Ministerio de Fomento y el Ayuntamiento para la cesión de la titularidad de la vía, así como de los tramos urbanos de otras vías hasta entonces dependientes del Ministerio. Esta cesión permitió al Ayuntamiento comenzar a ejecutar ese mismo año el proyecto Madrid Calle 30, nombre con el que desde entonces también se denomina la empresa mixta que gestiona la vía. Esta obra de soterramiento de una vía de circunvalación, que pretendía compararse con la construcción del Big Dig (Central Artery/Tunnel Project) en Boston. Ha sido un proyecto que ha requerido del liderazgo político de la Alcaldía, con protestas de movimientos ciudadanos y de otras instancias de Gobierno, como el Ministerio de Medio Ambiente y el Parlamento Europeo que criticaron las maniobras por evitar en primer término el Estudio de Impacto Ambiental.

Siguiendo con las infraestructuras, el gobierno nacional impulsa en 2006 la construcción de una nueva terminal, que incluye la T4 y su edificio satélite, el T4S, con más de $750.000 \mathrm{~m}^{2}$, lo que permite la obtención de Madrid-Barajas de la condición de hub mundial.

También existe una gran actividad relacionada con el impulso de programas muy diversos. En el periodo se aprueban varios planes específicos, entre otros: Plan Director Movilidad Ciclista 2008; Plan de Uso Sostenible de la Energía 2005; Plan Madrid de Convivencia Social e Intercultural 2004; Plan General de Cooperación al Desarrollo 2009-2012; Plan Municipal de Atención a la Infancia y Adolescencia 2005, o Plan de Seguridad Vial 2007-2011.

En esta fase, la participación de las asociaciones de vecinos y de otros colectivos tienen su espacio y se convierten en un actor de la producción de los espacios urbanos, mientras que el Ayuntamiento ejercerá un rol de intermediario más activo en el conflicto social. Así, se consideran por el ayuntamiento una propuesta de participación presupuestaria los planes especiales de inversiones y actuaciones en los distritos madrileños. Desde 2004 se han negociado y aprobado los de Latina, Carabanchel, Tetuán, San Blas, Vicálvaro, Usera, Villaverde, Puente de Vallecas y 
Villa de Vallecas. Se completan con Planes de Barrio y Planes de Equipamiento (para Las Tablas y Montecarmelo).

Estos planes pueden considerarse la continuación de los planes de inversiones en Usera y Villaverde y en Puente y Villa de Vallecas propuestos por la Federación Regional de Asociaciones de Vecinos de Madrid (FRAVM). En palabras de la Asociación de Vecinos de Madrid "este modelo no reproduce fielmente el ejemplo de lo que llamamos los «presupuestos participativos», pero bien nos gustaría que cundiera el ejemplo (tanto más entre ciertos «críticos» de los planes), toda vez que la participación, como la solidaridad, aunque se habla mucho de ellas, escasean por demás en estos tiempos" (Asociaciones de Vecinos de Madrid: 2006). Por el contrario, otras Asociaciones de Vecinos decidieron no participar y realizar un boicot a los Consejos Territoriales y Mesas de Diálogo (2006) por considerar que estaban manipuladas por el gobierno municipal. En todo caso, lo que nos interesa resaltar es que sea el poder local el interlocutor principal como receptor de las demandas o los órganos de participación creados por el gobierno municipal, en cualquier caso se devuelve al ayuntamiento a la esfera de lo político.

\section{La política urbana en la ciudad de Madrid: claves para su interpretación}

Hemos realizado un rápido recorrido por las acciones más significativas que se han impulsado con la intención de intervenir en las dimensiones urbanísticas, económicas, sociales o culturales de la ciudad de Madrid. Esta mirada nos permite proponer algunas claves para entender el camino seguido, cuáles han sido las decisiones que han convertido a la ciudad en lo que hoy es y quienes han sido sus protagonistas. La perspectiva también permite construir un relato más coherente del conjunto de acciones inconcretas y aparentemente inacabadas que son propias de las políticas públicas.

\subsection{Madrid en tránsito hacia la ciudad global}

Cuando los primeros ayuntamientos democráticos comienzan a trabajar a principios de los años 1980, el mundo era diferente. Todavía dominaba el modelo de desarrollo basado en la extensión del bienestar y en las políticas keynesianas; aunque existían ya voces críticas, todavía no se había cuestionado la necesidad de instituciones político-administrativas fuertes con gran capacidad de intervención. A pesar del aumento de las comunicaciones, cada unidad política tenía cierta capacidad de pensar de manera aislada en sus opciones futuras y, además, la experiencia de otros países occidentales enseñaba algunas claves de lo que podría hacerse en el nivel local español. La suma de estos factores, y de algunos otros, hacía posible un mun- 
do urbano más diferenciado, en donde cada ciudad podía trabajar concentrada en sí misma.

En ese ambiente, la primera política urbana de Madrid busca construir un modelo propio de ciudad, partiendo de los problemas que existen y abriendo la discusión y las decisiones a los actores privados; el ejemplo más significativo es el Programa de Barrios en Remodelación. Este enfoque empieza a cambiar ya al final de la década de los ochenta. Entonces se observan las primeras reacciones en la acción pública de la crisis ideológica del modelo, en especial el cuestionamiento de la sostenibilidad económica del mismo, la crisis fiscal o la crítica a la ineficacia y tamaño que han adquirido las organizaciones político-administrativas. Se convoca un primer proceso de planificación estratégica y se crean los primeros entes autónomos para gestionar dimensiones importantes de la política urbana desde organizaciones nuevas, caso de la Empresa Municipal de la Vivienda.

El giro se acentúa en la fase gerencial de manera evidente. Se trata de dotar a la ciudad de los requerimientos que parecen de mayor interés para los decisores económicos internacionales: nuevos espacios para oficinas; red viaria moderna y amplia; excelentes infraestructuras para el transporte colectivo - especialmente aeropuerto-; centros logísticos de apoyo o parques tecnológicos de última generación.

El último de los impulsos se da en la fase de la ciudad global. En este caso los decisiones no sólo quieren que la ciudad resulte atractiva para el capital internacional, quieren que Madrid forme parte de manera clara de las listas de ciudades de importancia planetaria, que esté presente en los rankings de importancia urbana, que forme parte del grupo de ciudades nodo, ciudades globales, núcleos de decisión planetaria... Para ello se crea un organismo cuya función sea el seguimiento continuado de los numerosos rankings y clasificaciones de ciudades existentes y el desarrollo de la diplomacia urbana. Y, ¿cuál podría ser el objetivo de un empeño como éste?

Encontramos dos razones que se superponen. La primera es que algunos casos han demostrado que no sólo son exitosas las ciudades que facilitan los requerimientos infraestructurales y de servicios al empresariado internacional; otras han llegado a ser espacios de interés para aquellos a través de estrategias diferentes, como la cultura. Esto significa que, no habiendo un solo camino, es necesario estar alerta para sumarse a varios. La segunda entronca con el siguiente punto y es que la lógica de las ciudades globales y las prácticas neoliberales son dos caras de una misma moneda. Vainer (2000) explica de manera convincente y gráfica que la política urbana neoliberal considera la ciudad como una mercancía para ser comercializada y que el gobierno local se comporta como una empresa privada gestionando la ciudad. El aglutinante que cohesiona a la opinión pública es el fortalecimiento del orgullo local, lo que el autor denomina la patria urbana. Creemos que la lógica de los rankings y clasificaciones fortalece también esa dimensión. 
Figura 1. Evolución de una política urbana endógena a una lógica global

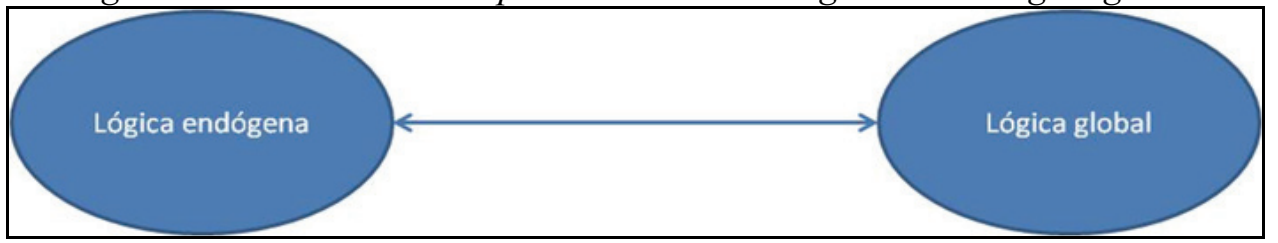

Fuente: elaboración propia.

En la Figura 1 se expresa de manera gráfica la idea de un continuum en donde, de manera ideal, podríamos encontrar, en un extremo, una política urbana concentrada en la lógica interna de la propia ciudad $y$, en el otro, una política urbana centrada en la lógica global.

\subsection{El fortalecimiento de las politicas neoliberales de Madrid}

Observando las acciones implantadas, se detecta un giro desde una posición en donde un gobierno más intervencionista despliega políticas sociales para requilibrar el bienestar de la ciudad, a una nueva situación en donde una concepción neoliberal supone un repliegue público y una idea más competitiva del papel de las ciudades y, en este contexto, de Madrid.

Es posible caracterizar el estilo de gobierno de las diferentes fases calificando el papel que asumen los gobiernos locales según los programas realmente impulsados (Cuadro 3).

Cuadro 3. Estilos de gobierno en las cuatro fases de política urbana en Madrid

\begin{tabular}{|l|l|l|l|l|}
\hline & Franquismo & $\begin{array}{c}\text { Democratiza- } \\
\text { ción }\end{array}$ & Gerencialismo & Globalización \\
\hline $\begin{array}{l}\text { Estilo de } \\
\text { liderazgo del } \\
\text { gobierno local }\end{array}$ & Burócratas & Políticos & Gestores & Empresarios \\
\hline
\end{tabular}

Fuente: elaboración propia.

A pesar de que el giro resulta evidente, sería necesario profundizar en el contenido e impacto real de lo hecho, ya que la denominación de los programas y descripción de sus objetivos genera algunas dudas. Habría que observar si las acciones realmente implantadas responden o no a las características de las estrategias urbanas neoliberales (Peck y Tickell, 2002): 
- Enfoque "crecer primero" para el desarrollo urbano, supeditando el bienestar social al desarrollo económico.

- Consideración del mercado como el modelo ideal de toma de decisiones y distribución.

- Apoyo a procesos privatizadores y desregularizadores.

- Posición de alerta hacia el exterior para hallar oportunidades, buenas prácticas y potenciales competidores en un mundo en lucha por recursos globales.

- Recurso a un limitado rango de políticas urbanas.

- Enfrentamiento de problemas a través de formas autoritarias de gobernanza.

Figura 2. Evolución de una política urbana endógena a una lógica global

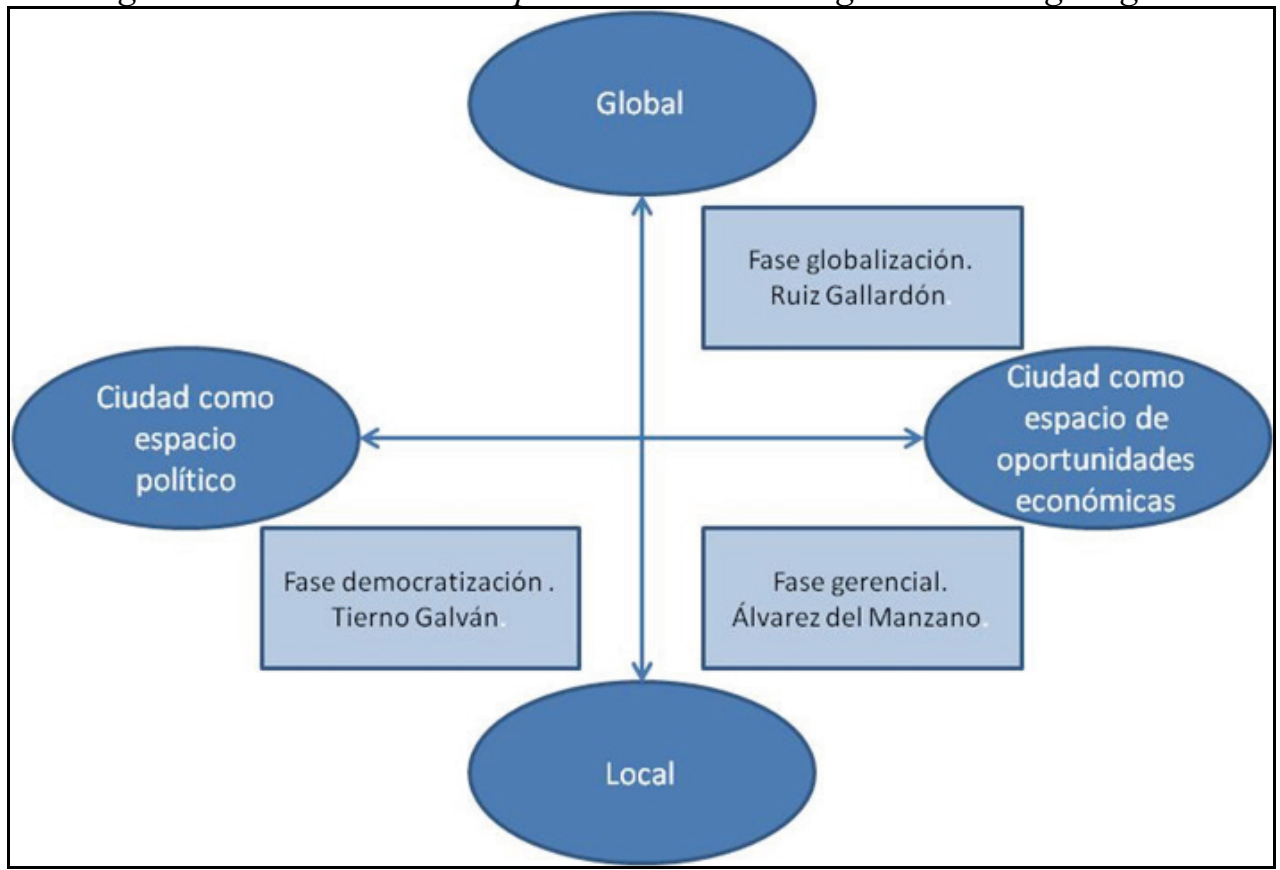

Fuente: elaboración propia.

Del análisis realizado resulta posible combinar dos claves para interpretar la política urbana. La primera, descrita en el epígrafe anterior, apunta que es posible localizar en el discurso de las políticas urbanas una visión más endógena o local, frente a una visión más externa o global. Una segunda clave para comprender el cauce de acción impulsado es considerar el peso que tiene el discurso de representar la ciudad como un espacio político, en un extremo, frente a representar la ciudad como un espacio de oportunidades económicas, en el otro. La representación gráfica del cruce de ambas propuestas (Figura 2) nos permite observar cómo los diferentes 
gobiernos de la ciudad de Madrid, aún siendo del mismo partido político, ocupan distintas posiciones en estos ejes.

Esta idea de la variedad de propuestas neoliberales ya ha sido expresada respecto de otras ciudades "a lo largo de este periodo ha ocurrido una marcada urbanización del neoliberalismo, a medida que las ciudades han devenido metas estratégicas y terrenos de prueba para una cada vez más amplia gama de experimentos de políticas neoliberales, innovaciones institucionales y proyectos políticos" (Theodore, Peck y Brenneriy, 2009: 10).

\subsection{Las contradicciones de la capitalidad}

Madrid presenta muchas de sus peculiaridades asociadas a su condición de ciudad capital. Ser el locus político central de la escala política nacional supone algunos condicionantes previos relacionados con la política urbana que se diseña e implanta para la misma: a) la capital tiene una altísima densidad institucional, aglutinando en su espacio instituciones públicas y de gobierno de diferentes escalas, lo que resta protagonismo a las instituciones locales; b) como consecuencia de lo anterior, se desdibuja el rol que el gobierno local ha tenido en su construcción o producción (Lefebvre, 1976) en la medida en que históricamente otras escalas de poder han producido y transformado la ciudad, y c) pero, a cambio, esto facilita los procesos de visibilidad y reconocimiento internacional.

La conclusión es que la capitalidad ha sido un factor positivo para algunas cuestiones (fundamentalmente relacionadas con la inversión pública en infraestructuras para la mejora del transporte público y privado), pero negativo para otras (en especial, en lo relacionado con el interés último de los actores políticos que prestan sus servicios para la ciudad que, como es claro en la evolución y presente de la ciudad, la ven como una plataforma de promoción a otros niveles de gobierno). Esto nos lleva de nuevo a las investigaciones que tratan de conectar la historia de la ciudad con la posición en el presente (Sassen, 2007).

Madrid, a diferencia de otras ciudades, comenzó a recuperarse económicamente de las crisis fordistas gracias a su posición privilegiada de capital y, por tanto, comienza el cambio de políticas cuando ya había salido beneficiada de las nuevas necesidades de la fase de acumulación, a saber: la concentración del sector financiero (Valenzuela, 1990: 20) y de la inversión extranjera. Esto hace que en esta ciudad el cambio de una a otra fase no se explica por "contexto económico regresivo con cambio de paradigma productivo hacia modelos postindustriales con efectos devastadores en áreas urbanas y metropolitanas; la crisis industrial y de empleo; la extensión de la desigualdad social y la creciente complejidad de las estructuras urbanas" (Brugué y Gomá, 1998: 562), sino por otros factores. 
Por último, las políticas impulsadas por otras escalas de gobierno influyen en la producción del espacio urbano y condicionan las actuaciones del gobierno municipal. Esto es especialmente claro en el caso de la capital. Sin embargo, será un tema más relacionado con el carácter del liderazgo de los actores lo que acabe generando los diferentes modelos de ciudad.

\subsection{Contrapesos en los liderazgos de Madrid}

Las posibilidades de comparar políticas urbanas se complican en la medida en que las historias socio-espaciales de las ciudades son disímiles y los liderazgos políticos también. En el caso de Madrid, la producción de políticas públicas urbanas en las últimas décadas está claramente vinculada a los liderazgos de los presidentes de la Comunidad Autónoma de Madrid y de los alcaldes, independientemente de los vínculos partidistas.

El liderazgo es difícil de medir, sin embargo es posible determinar si el protagonista de las acciones más importantes de cada fase pertenece al gobierno local o a otros niveles. En las cuatro fases descritas domina, como hemos visto, una escala espacial que se convierte en el referente básico de las políticas urbanas y, también en cada fase, se impone un estilo de gobierno diferenciado.

Cuadro 4. Referencias y estilos de gobierno en las cuatro fases de política urbana en Madrid

\begin{tabular}{|l|c|c|c|c|}
\hline & Franquismo & $\begin{array}{c}\text { Democratiza- } \\
\text { ción }\end{array}$ & Gerencialismo & Globalización \\
\hline $\begin{array}{l}\text { Escala espa- } \\
\text { cial que se } \\
\text { convierte en } \\
\text { referente de } \\
\text { las políticas } \\
\text { urbanas }\end{array}$ & España & Madrid & $\begin{array}{c}\text { Comunidad } \\
\text { Autónoma }\end{array}$ & Mundo \\
\hline $\begin{array}{l}\text { Estilo de } \\
\text { liderazgo del } \\
\text { gobierno local }\end{array}$ & Burócratas & Políticos & Gestores & Empresarios \\
\hline
\end{tabular}

Fuente: elaboración propia.

En la primera fase de gobiernos democráticos existía un liderazgo político claro, con una única competencia institucional directa, la del Gobierno Central; pero las acciones más significativas del periodo para la ciudad son impulsadas por el nivel local. 
En la siguiente fase, existe un gobierno local tecnocrático que convive con una Comunidad Autónoma dirigida por políticos con marcado liderazgo político. Esto supone que las políticas urbanas más significativas de la etapa, son impulsadas por la Comunidad Autónoma. Siempre la intervención del gobierno municipal está muy claramente imbricada con las políticas públicas de otras escalas (europea, nacional y regional), pero durante esta fase el protagonismo, la iniciativa del diseño de las políticas y programas recae en la Comunidad Autónoma, en detrimento del municipio.

En la última, el liderazgo político es claro, con un liderazgo también fuerte en la Comunidad Autónoma. Pero, para la política urbana, el Alcalde y el nivel municipal son los protagonistas absolutos.

A esta información podemos añadir un nuevo elemento: la concepción de la ciudad como un espacio político con personalidad propia o como un espacio de gestión que puede aprovechar programas de otras instancias (Cuadro 5).

Cuadro 5. Origen de las políticas más significativas, referencias y estilos de gobierno en las cuatro fases de política urbana en Madrid

\begin{tabular}{|l|l|l|l|l|}
\hline & Franquismo & $\begin{array}{c}\text { Democratiza- } \\
\text { ción }\end{array}$ & Gerencialismo & Globalización \\
\hline $\begin{array}{l}\text { Origen de las } \\
\text { políticas más } \\
\text { significativas }\end{array}$ & $\begin{array}{l}\text { Gobierno } \\
\text { Central }\end{array}$ & Gobierno Local & $\begin{array}{l}\text { Comunidad } \\
\text { Autónoma }\end{array}$ & Gobierno Local \\
\hline $\begin{array}{l}\text { Escala espacial } \\
\text { que se convier- } \\
\text { te en referente } \\
\text { de las politicas } \\
\text { urbanas }\end{array}$ & España & Madrid & $\begin{array}{l}\text { Comunidad } \\
\text { Autónoma }\end{array}$ & Mundo \\
\hline $\begin{array}{l}\text { Estilo de } \\
\text { liderazgo del } \\
\text { gobierno local }\end{array}$ & Burócratas & Políticos & Gestores & Empresarios \\
\hline
\end{tabular}

Fuente: elaboración propia.

Podemos representar gráficamente la posición que asume cada uno de los gobiernos locales democráticos analizados teniendo en cuenta dos ejes: un eje que refleja la visión local o global de la ciudad y otro eje en donde situar si las políticas urbanas más significativas reflejan un protagonismo local o de la Comunidad Autónoma. Como se observa en la Figura 3, la representación permite comprender mejor las diferencias que existen entre políticas urbanas impulsadas por gobiernos del mismo partido. 
Figura 3. Protagonismo del gobierno local en Madrid y lógica imperante

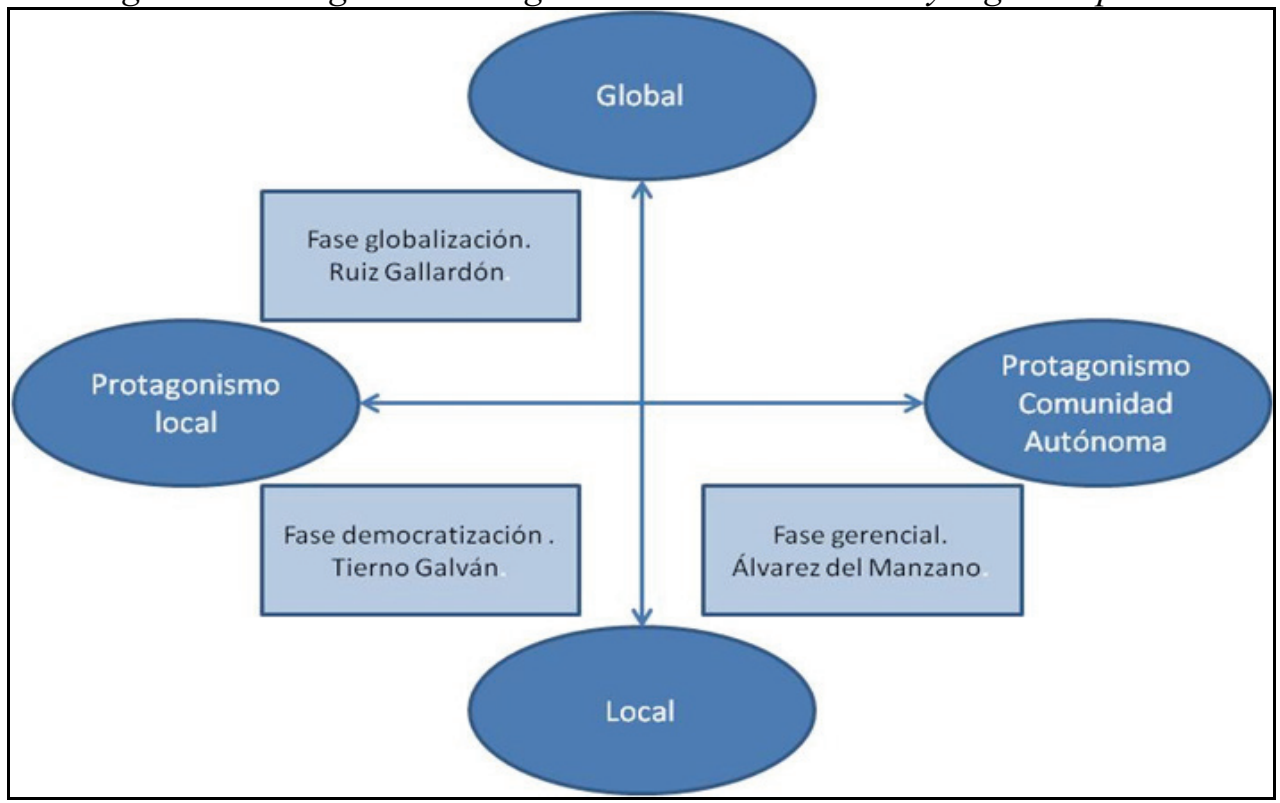

Fuente: elaboración propia.

\section{Conclusiones y algunas preguntas para el futuro}

Nuestra conclusión general es que si la ciudad tiene un gobierno con un claro liderazgo político, la política urbana es básicamente local, perdiendo protagonismo cualquier otro nivel de gobierno. Pero, cuando el gobierno local tiene un acento más gerencial, entonces deja espacio al nivel político superior para liderar la acción política.

A pesar de tener una visión más detallada de cómo ha sido la política urbana de la ciudad de Madrid, quedan aún algunas cuestiones que podrían ser objeto de reflexión.

En especial creemos interesante analizar los espacios de representación alternativos o contrahegemónicos al intentar clasificar las políticas urbanas, de manera que podamos conocer la relación entre ambos procesos, su interrelación e influencia mutua. Asimismo, conocer las posibilidades que la ciudadanía tiene para intervenir en la producción del espacio urbano, bien para aprobar las otras dinámicas (económica y política) o bien generar espacios diferentes y contrarios a las dinámicas producidas.

O, finalmente, observar si existen "espacios de representación", en la línea del término Lefevbre (1976), para mostrar cómo la ciudad es producida alternativa por 
nuevas imaginaciones espaciales que pretenden romper y contrarrestar los efectos de las otras formas de producir el espacio.

\section{Bibliografía}

Alguacil, J.; de la Fuente, R. et al. (2012) “Madrid”, en M. Iglesias, M. Martí Costa, J. Subirats y M. Tomás (eds.) Políticas urbanas en España. Barcelona: Icaria.

Asociación de vecinos de Madrid (2006) Planes especiales inversiones y actuaciones a enero de 2006 [URL: http://aavvmadrid.org/index.php/Areas-deTrabajo/Planes-Especiales-de-Inversiones-y-Actuaciones/. Consultado el 1 de julio de 2012].

Ayuntamiento de Madrid (2012) "Cañada Real Galiana" [URL: http://www.madrid.es/portales/munimadrid/es/Inicio/Ayuntamiento/Urbanismoe-Infraestructuras/. Consultado el 14 de julio de 2012].

Brenner, N. (2003) "La formación de la ciudad global y el re-escalamiento del espacio del Estado en la Europa Occidental post-fordista". EURE [en línea], vol. 29, $\mathrm{n}^{\circ} .86,5-35$. [URL: <http://www.eure.cl/numero/la-formacion-de-la-ciudadglobal-y-el-re-escalamiento-del-espacio-del-estado-en-la-europa-occidentalpost-fordista/>. Consultado el 17 de Julio de 2011].

Brugué, Q. y Gomà, R. (1998) "Gobierno local, ciudad y política urbana". Estudios Demográficos y Urbanos, vol. 13, $\mathrm{n}^{\mathrm{o}} .3,561-583$.

Castells, M. (2002) "Local and global: Cities in the network society". Tijdschrift voor Economische en Sociale Geografie, vol. 93, $\mathrm{n}^{\circ} .5,548-558$.

Castells, M. (1989) The Informational City. Oxford: Blackwell.

Cochrane, A. (2007) Understanding Urban Policy. Oxford: Blackwell Publishing.

Crot, L. (2010) "Transnational urban policies: «relocating» Spanish and Brazilian models of urban planning in Buenos Aires". Urban Research \& Practice, vol. 3, $\mathrm{n}^{\mathrm{o}} .2,119-137$.

De Santiago Rodríguez, E. (2007) "Madrid, «ciudad única». Pautas y lógicas espaciales recientes en la región madrileña: las grandes transformaciones estructurales; el despliegue del nuevo «paradigma único» en la región urbana de Madrid". Urban, vol. 12, $\mathrm{n}^{\mathrm{o}} .2,8-33$.

Friedmann, J., y Wolff, G. (1982) "World city formation: An agenda for research and action". International Journal of Urban and Regional Research, vol. 3, $\mathrm{n}^{\mathrm{o}} .2$, 309-344.

Harvey, D. (2005) A brief history of neoliberalism. Oxford: Oxford University Press.

Iglesias, M.; Martí Costa, M.; Subirats, J., y Tomás, M. (eds.) (2012) Políticas urbanas en España. Barcelona: Icaria.

Jenkins, W. I. (1978) Policy Analysis: A Political and Administrative Perspective. Oxford: Martin Robertson. 
Larner, W. (2000) "Theorising Neoliberalism: Policy, ideology, governmentalism". Studies in Political Economy, vol. 63, Autumn, 5-26.

Lefebvre, H. (1976) "Reflections on the politics of space". Antipode, vol. 8, n". 2, 30-37.

López de Lucio, R. (2000) "Madrid 1979-1999. Perfiles de una transformación urbana desconocida". Urban, vol. 4, nº. 9, 106-123.

Mény, I., y Thoenig, J. C. (1992) Las políticas públicas. Barcelona: Ariel.

Oficina de Estrategia y Acción Internacional (2008) Madrid Global. Madrid: Ayuntamiento de Madrid.

Parsons, W. (1995) Public Policy. Cheltenham: Edward Elgar.

Peck, J., y Tickell, A. (2002) "Neoliberalizing Space". Antipode, vol. 34, n. 3, 480404.

PROMADRID (1993) Madrid Futuro: Plan Estratégico de Madrid. Madrid: Ayuntamiento de Madrid.

Sassen, S. (2007) "El reposicionamiento de las ciudades y regiones urbanas en una economía global: ampliando las opciones de políticas y gobernanza". EURE [en línea], vol. 33, $\mathrm{n}^{\circ}$. 100, 9-34. [URL: <http://www.eure.cl/numero/elreposicionamiento-de-las-ciudades-y-regiones-urbanas-en-una-economia-globalampliando-las-opciones-de-politicas-y-gobernanza/>. Consultado el 17 de Julio de 2011].

Sassen, S. (1998) "Ciudades en la economía global: enfoques teóricos y metodológicos". EURE [en línea], vol. 24, no. 71, 5-25. [URL: $<$ http://www.eure.cl/numero/ciudades-en-la-economia-global-enfoques-teoricosy-metodologicos/>. Consultado el 17 de Julio de 2011].

Sassen, S. (1991) The global city. New York, London, Tokyo. Princeton: Princeton University Press.

Subirats, J. et al. (2008) Análisis y Gestión de Políticas Públicas. Barcelona: Ariel.

Swyngedouw, E. (2004) "Globalisation or «Glocalisation»? Networks, Territories and Rescaling". Cambridge Review of International Affairs, vol. 17, $\mathrm{n}^{\circ} .1,25-48$.

Theodore, M.; Peck, J., y Brenner, N. (2009) "Urbanismo neoliberal: la ciudad y el imperio de los mercados". Temas Sociales, ${ }^{\circ}$. 66, 1-11. [URL: $<$ http://www.sitiosur.cl/publicacionescatalogodetalle.php?PID=3532\#descargar/

$>$ Consultado el 16 de mayo de 2012].

Vainer, C. (2000) "Patria, empresa y mercadería", en O. Arantes, C. Vainer y E. Maricato: A cidade do pensamiento único. Desmanchando consensos. Petrópolis: Ed. Vozes $\left(2^{\mathrm{a}}\right.$ ed).

Valenzuela Rubio, M. (1990) "La comunidad de Madrid y la construcción del territorio metropolitano". Boletín de la Asociación de Geógrafos, $\mathrm{n}^{\circ}$. 11, 15-50. 